\title{
Analysis of wheat microspore embryogenesis induction by transcriptome and small RNA sequencing using the highly responsive cultivar "Svilena"
}

Felix Seifert ${ }^{1}$, Sandra Bössow ${ }^{2}$, Jochen Kumlehn ${ }^{3}$, Heike Gnad ${ }^{2^{*}}$ and Stefan Scholten ${ }^{1,4^{*}}$

\begin{abstract}
Background: Microspore embryogenesis describes a stress-induced reprogramming of immature male plant gametophytes to develop into embryo-like structures, which can be regenerated into doubled haploid plants after whole genome reduplication. This mechanism is of high interest for both research as well as plant breeding. The objective of this study was to characterize transcriptional changes and regulatory relationships in early stages of cold stress-induced wheat microspore embryogenesis by transcriptome and small RNA sequencing using a highly responsive cultivar.

Results: Transcriptome and small RNA sequencing was performed in a staged time-course to analyze wheat microspore embryogenesis induction. The analyzed stages were freshly harvested, untreated uninucleate microspores and the two following stages from in vitro anther culture: directly after induction by cold-stress treatment and microspores undergoing the first nuclear divisions. A de novo transcriptome assembly resulted in 29,388 contigs distributing to 20,224 putative transcripts of which 9,305 are not covered by public wheat cDNAs. Differentially expressed transcripts and small RNAs were identified for the stage transitions highlighting various processes as well as specific genes to be involved in microspore embryogenesis induction.
\end{abstract}

Conclusion: This study establishes a comprehensive functional genomics resource for wheat microspore embryogenesis induction and initial understanding of molecular mechanisms involved. A large set of putative transcripts presumably specific for microspore embryogenesis induction as well as contributing processes and specific genes were identified. The results allow for a first insight in regulatory roles of small RNAs in the reprogramming of microspores towards an embryogenic cell fate.

Keywords: Microspore embryogenesis induction, Transcriptome, Small RNA, RNA-seq, sRNA-seq, Epigenetics, Wheat

\section{Background}

Microspore embryogenesis or androgenesis involves the competence of the immature male gametophyte to switch from gametophytic to embryonic developmental cell fate through an inductive treatment prior to or at the initiation of anther or microspore culture [1]. It is an

\footnotetext{
* Correspondence: gnad@saaten-union-biotec.com; s.scholten@unihohenheim.de

${ }^{2}$ Saaten-Union Biotec GmbH, Am Schwabenplan 6, 06466 Seeland, OT Gatersleben, Germany

${ }^{1}$ Developmental Biology, Biocenter Klein Flottbek, University of Hamburg, Ohnhorststrasse 18, 22609 Hamburg, Germany

Full list of author information is available at the end of the article
}

illustrative example and model for developmental plasticity and cell fate decisions in plants and an important tool in research and plant breeding for the generation of doubled haploid plants [2]. Double haploid technology is widely employed in breeding programs of many crop species for its possibility to quickly generate diverse recombinant, yet genetically fixed individuals [3]. While bread wheat (Triticum aestivum) is one of the globally most important crops that amount for $20 \%$ of the human calorie consumption [4], most of its cultivars are highly recalcitrant to microspore embryogenesis. Functional genetic studies to dissect tissue culture responses are first steps in overcoming these 
limitations to enhance bread wheat breeding eventually. Numerous microarray based gene expression studies were conducted to elucidate the major switches from gametophytic to embryonic development in various plants $[5,6]$. These experiments revealed large scale patterns in the reprogramming of microspores to embryogenic tissues, which indicated a reset of the transcriptional and translational profiles to arrest gametophytic development [7]. Nevertheless, those studies were limited by the particular microarray platform used, which likely did not cover all genes specifically expressed in the reprogramming process of microspore embryogenesis, due to a biased microarray design to transcripts expressed primarily in vegetative tissues. The advent of high throughput transcriptome analysis allows for an unlimited global analysis of expressed transcripts. Thus we performed a transcriptome sequencing (RNA-seq) study analyzing three early stages around microspore embryogenesis induction, to elucidate transcriptomic changes of two major transitions of embryogenesis induction leading to first nuclear divisions. Recently, epigenetic mechanisms were proposed to regulate the transition from gametophytic to embryogenic cell fate [8-10]. Small non-conding RNAs (sRNAs) were shown to be involved in the remodulation of the epigenetic landscape and transcript levels through different mechanisms [11], and thus are putatively potent regulators. We performed sRNA sequencing (sRNA-seq) of the same time-series as for RNA-seq, to allow for a comprehensive analysis of both sRNA and transcriptome expression changes and for the discovery of putative regulatory relationships. Our study provides the first deep sequencing-based resource for functional genomics research of microspore embryogenesis induction in wheat.

\section{Results and discussion}

\section{Development of microspores and sampling}

Donor plants of the winter wheat cultivar "Svilena", which is highly responsive to stress-induced microspore embryogenesis [12], were used for anther culture as described by Rubtsova et al. (2013) [13]. Microspores were sampled at three stages: a) freshly harvested microspores at their late, uninucleate highly vacuolated stage (S1), b) microspores after 10 days of cold pre-treatment exhibiting a star-like structure (S2) and c) microspores undergoing early nuclear division (S3) based on visual assessment (Fig. 1). These visually distinct developmental phases in microspore embryogenesis induction represent crucial stages in the acquisition of embryogenic potential, which were elucidated in various cytological studies $[1,7,14]$. It has been shown that microspores, before, or immature pollen, directly after pollen mitosis $\mathrm{I}$, are most responsive for stress treatment-induced embryogenic development. The first effect after stress treatment is a rearrangement of the cytoskeleton resulting in the re-localisation of the nucleus to the center of the cell. The nucleus is surrounded by cytoplasmic strands and thus a star-like structure is formed by this process, which was suggested to be the first sign of embryogenic induction $[2,15]$. The manual sorting procedure that we applied for RNA-seq facilitates a very high homogeneity and thus a stage-specific analysis of the pooled microspores as well as an exclusion of injured or dead cells. Due to higher RNA amount requirements for sRNAseq, a gradient centrifugation-based isolation was performed, which delivers the required cell numbers at the cost of slightly reduced population homogeneity. To control for batch-to-batch variations, donor material for all microspore isolations for RNA-seq and sRNA-seq were cultured until plant regeneration. In

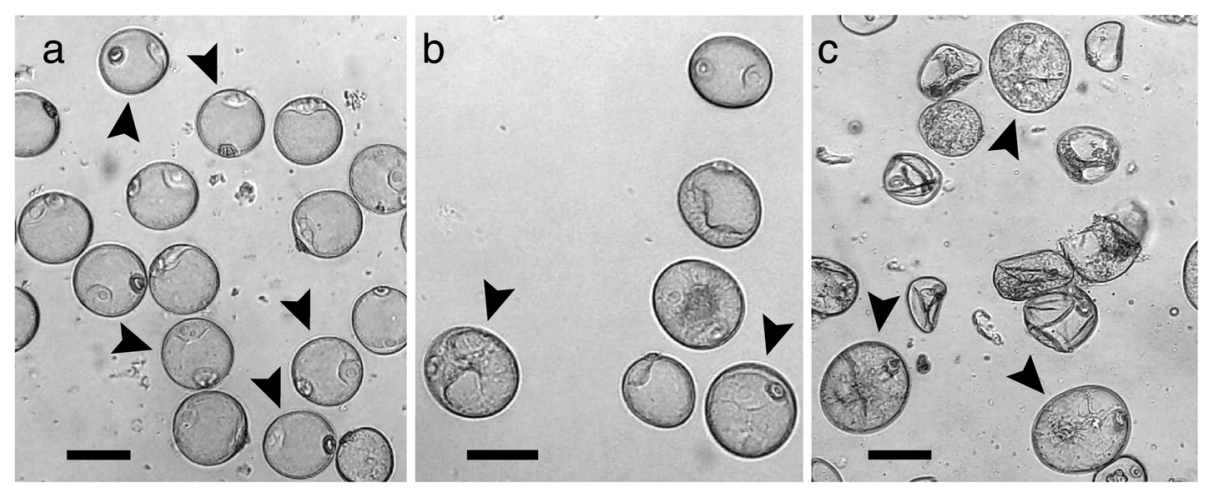

Fig. 1 Microspore development stages sampled for RNA sequencing analysis. Brightfield micrographs of representative samples from three microspore stages. All bars represent $20 \mu \mathrm{m}$. Arrowheads point to cells with morphological characteristics that meet our criteria for manual cell selection. a Untreated vacuolated microspores at uninucleate stage (S1); manually selected microspores were characterized by a large central vacuole and a clear cytoplasm. b Microspores with star-like structure after 10-days cold stress pre-treatment (S2); microspores are slightly enlarged after stress induction, the vegetative nucleus migrates into the center of the cell, the cytoplasm becomes structured and shows cytoplasmic strands, the so-called "star-like structure". c Microspores undergoing first nuclear division (S3); the vegetative nucleus is centrally located and has divided 
either case the high regeneration frequency was equivalent to the usually observed response for the cultivar "Svilena".

\section{Transcriptome sequencing}

RNA-seq allows an unrestricted and global analysis of gene expression as well as the identification of unknown transcripts. To facilitate a comprehensive overview of gene expression through acquisition of embryogenic potential of microspores, we sequenced the samples in biological triplicates for each stage. All libraries were indexed with unique nucleic acid identifiers and $50 \mathrm{bp}$ single end reads were sequenced on an Illumina HiSeq 2000 sequencer. In total, 608,233,335 clean RNA-seq reads were generated, with individual libraries covering 55.6 Mio. to 75.6 Mio. reads (see Table 1).

\section{De novo transcriptome assembly and annotation}

A de novo transcriptome assembly using the Trinity de novo assembler [16] was performed based on the RNAseq reads of all stages and replicates and resulted in 29,388 contigs with an average length of $417.87 \mathrm{bp}$. The size distribution of the contigs is shown in Fig. 2a. Our approach allows for an expression comparison as well as a functional annotation for the identification of important gene functions in microspore embryogenesis induction. We did not pursue resolving the homeologs or isoforms, this would have required a higher sequencing depth as well as longer and paired end reads. A BLASTx mapping resulted in 18,344 (62.42\%) contigs with homology to protein sequences in the NCBI nr database. The majority of contigs exhibits the highest sequence homology with Aegilops tauschii and Triticum urartu, known to be the diploid progenitors for the wheat $\mathrm{A}$ and D genome, respectively [17], followed by other grass species (Fig. 2b). This indicates wheat specific sequencing results without contamination and an effective de novo

Table 1 Summary of RNA-seq/sRNA-seq data

\begin{tabular}{|c|c|c|c|c|}
\hline \multirow[b]{2}{*}{$\begin{array}{l}\text { Sample } \\
\text { replicate }\end{array}$} & \multicolumn{2}{|c|}{ RNA-seq data } & \multicolumn{2}{|c|}{ sRNA-seq data (18 to $28-n t)$} \\
\hline & $\begin{array}{l}\text { Trimmed } \\
\text { reads }\end{array}$ & $\begin{array}{l}\text { Uniquely mapping } \\
\text { reads to de novo } \\
\text { transcriptome [\%] }\end{array}$ & $\begin{array}{l}\text { Trimmed } \\
\text { reads }\end{array}$ & Distinct reads \\
\hline S1a & $70,216,602$ & 39.442 & $10,425,301$ & $1,813,927$ \\
\hline$S 1 b$ & $75,697,405$ & 38.307 & $10,247,910$ & $2,332,967$ \\
\hline S1c & $55,646,245$ & 34.298 & $9,892,496$ & $2,983,585$ \\
\hline S2a & $66,434,325$ & 42.742 & $10,707,291$ & $3,092,207$ \\
\hline $\mathrm{S} 2 \mathrm{~b}$ & $66,122,827$ & 43.012 & $9,718,833$ & $2,572,135$ \\
\hline S2C & $73,711,917$ & 42.240 & $10,154,174$ & $3,174,185$ \\
\hline S3a & $71,321,690$ & 44.759 & $10,789,357$ & $2,908,387$ \\
\hline S3b & $70,400,647$ & 50.624 & $10,664,717$ & $3,063,376$ \\
\hline S3C & $58,681,677$ & 40.775 & $9,939,297$ & $4,006,577$ \\
\hline
\end{tabular}

assembly resulting in high homology to known monocot transcripts.

We annotated the contigs by assigning gene ontology (GO) terms via Blast2GO [18] and Trinotate [19]. This annotation resulted in 13,553 (46.12 \%) contigs with a homology-based annotation, with on average 7.41 GO terms per contig. Mapping of the contigs to known wheat cDNA sequences (ensembl release 26) [20] resulted in 10,919 cDNAs covered by contigs from the RNA-seq de novo assembly. A subset of 5,139 wheat cDNAs were covered by multiple contigs (on average 2.78 contigs per cDNA) most likely due to fragmented assembly of the short reads. The restructuring of the contigs to transcripts based on wheat cDNA sequences revealed 20,224 transcripts covered by our de novo assembly. The contig assignment to transcripts is listed in Additional file 1: Table S1. This restructured assembly contains 9,305 new transcripts not covered by known wheat cDNAs from ensembl release 26 [20], presumably because the specific cell-types, developmental stages and induction conditions used in the present study were not covered by previous sequencing efforts. Our dataset thus provides a valuable resource for the analysis of microspore embryogenesis. 3,206 (32.21 \%) of the new transcripts could be annotated by BLASTx mapping. The top hits from BLASTx for contigs attributed to restructured transcripts are shown in Additional file 2: Table S2. After the restructuring of contigs to transcripts, a GO annotation could be derived for 8,527 (42.16\%) of all transcripts including 996 transcripts not covered by wheat cDNAs (Additional file 3: Table S3). The GO annotation resulted in a large number of transcripts with biological processes related to response to stress and abiotic stimulus, which is most likely caused by the cold-stress treatment for microspore induction. Other main biological processes covered are cellular component organization, post-embryonic development, cell cycle, cell differentiation, embryo development and epigenetic regulation of gene expression (Table 2), which might be related to the developmental shift from gametophytic to embryogenic cell fate. The complete list of GO terms for all categories is shown in Additional file 4: Table S4.

\section{Expression analysis}

The expression levels of all transcripts were estimated based on uniquely mapping reads to the de novo assembled transcriptome (see Table 1). To allow for a comparison of replicates and stages the expression values were quantile normalized and scaled to one million quantile normalized reads per library (rpmqn). Correlation based clustering revealed that the expression values between the replicates exhibited a high similarity for each of the three specific stages and a clear separation from the other two stages (Fig. 2c). This clearly indicates that the manually sorted cells represent 


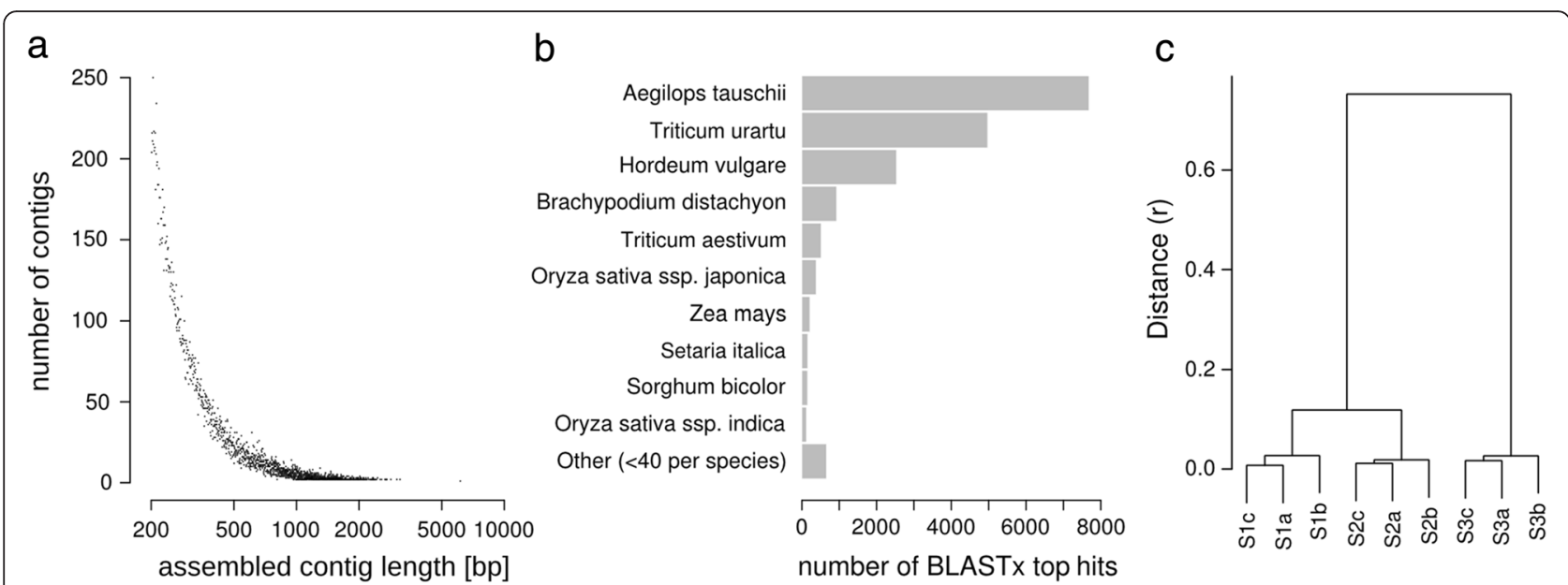

Fig. 2 Results from RNA-seq transcriptome assembly and expression analysis. a Size distribution of contigs assembled from RNA-seq reads of all replicates of the three microspore stages using the Trinity assembler. $\mathbf{b}$ Species distribution for BLASTx top hits of RNA-seq assembled contigs against the NCBI nr database. c Correlation-based clustering analysis for RNA-seq transcript expression values between the replicates of all microspore stages

uniform samples of developmentally distinct stages. Additionally we observed a much higher overall similarity between the transcriptomes of the stages $\mathrm{S} 1$ and S2 than between the first two stages and S3 (Fig. 2c). This result suggests, that the stress treatment causes few but drastic changes that direct to a large-scale reprogramming in the following transition.

For the analysis of stage specific transcription, we regarded transcripts with an expression of at least 1 rpmqn in all three replicates of at least one of the three stages as expressed. These thresholds revealed 14,792 $(73.14 \%)$ transcripts to be expressed in S1, an increase to 15,026 (74.3 \%) expressed transcripts in S2 followed by a decrease to 13,927 (68.86 \%) expressed transcripts in S3, respectively. The overlap of transcripts exclusively expressed in the stages S1 and S2 is 2,439 (12.06 \%) transcripts, but only $455(2.25 \%)$ transcripts were exclusively expressed in the stages S2 and S3 (see Fig. 3). A core set of 11,765 (58.17\%) transcripts was expressed in all three stages. The differing sets of expressed transcripts reflect the change of developmental fate in the transcriptome. Microspores that eventually develop into embryos have been shown to undergo a step of dedifferentiation first, which is completed at the stage exhibiting a star-like structure [7]. We found 24, 7, and 666 transcripts to be exclusively expressed in S1, S2, and S3, respectively (see Fig. 3). The transcripts along with their BLASTx top hits are listed in Additional file 5: Table S5. Interestingly, transcripts exclusively expressed in S3 cover transcripts which are known to be involved in acquisition of embryogenic cell fate, like transcript_14378 and transcript_18369 with similarity to RWP-RK DOMAIN CONTAINING 1 (RKD1), a transcription factor involved in female gametogenesis and early embryogenesis identified from isolated wheat egg cells [21]. transcript_7306 with similarity to AINTEGUMENTA-like 5 (AIL5), an AP2like ethylene-responsive transcription factor, which is a homolog to $B A B Y B O O M(B B M)$ and known to confer embryonic identity to cells [22]. transcript_11677 exhibits similarity to HIGH-LEVEL EXPRESSION OF SUGAR-INDUCABLE GENE2-LIKE1 (HSL1), which was shown to be specifically and highly expressed in early embryogenesis. Its interaction with the HISTONE DEACETYLASE 19 (HDA19) results in epigenetic repression of seed maturation genes [23]. Another epigenetic component, exclusively expressed in S3, is transcript_12642 with similarity to SHOOTLESS2 (SHL2), an orthologue of the Arabidopsis RNA-dependent RNA polymerase 6, which was shown to be involved in shoot apical meristem formation during embryogenesis [24]. Additionally, the specific expression of transcript_13594 and transcript_20002 in S3, both with homology to the DNA (cytosine-5)-methyltransferase $1 A$ (MET1a), is in agreement with reported DNA methylation dynamics and MET1a-like gene expression changes during stress-induced microspore reprogramming [25]. Overall, the large number of transcripts with homologies to known embryogenesis related genes suggests that we have identified many more not yet uncovered genes related to wheat microspore embryogenesis induction.

\section{Analysis of differentially expressed transcripts}

The transitions between the stages S1 and S2 (in the following denoted as T1) as well as between S2 and S3 (named T2) represent pivotal steps in induction and reprogramming from gametophytic fate of the microspore into embryo formation [7]. The differential expression (DE) of transcripts was determined for all transcripts with 
Table 2 Number of transcripts covered by $\mathrm{GO}$ terms of $\mathrm{GO}$ category biological process $(n>=100)$

\begin{tabular}{|c|c|c|}
\hline GO term & GO description & $\begin{array}{l}\text { Number of } \\
\text { transcripts }\end{array}$ \\
\hline GO:0009987 & cellular process & 3037 \\
\hline GO:0009058 & biosynthetic process & 1457 \\
\hline GO:0006950 & response to stress & 1373 \\
\hline GO:0016043 & cellular component organization & 1364 \\
\hline GO:0006810 & transport & 1232 \\
\hline GO:0009056 & catabolic process & 1197 \\
\hline GO:0008152 & metabolic process & 1147 \\
\hline GO:0006139 & $\begin{array}{l}\text { nucleobase-containing compound } \\
\text { metabolic process }\end{array}$ & 1094 \\
\hline GO:0006464 & cellular protein modification process & 1094 \\
\hline GO:0009628 & response to abiotic stimulus & 913 \\
\hline GO:0005975 & carbohydrate metabolic process & 763 \\
\hline GO:0008150 & biological process & 757 \\
\hline GO:0006350 & transcription, DNA-templated & 701 \\
\hline GO:0007275 & multicellular organismal development & 699 \\
\hline GO:0019538 & protein metabolic process & 676 \\
\hline GO:0006259 & DNA metabolic process & 587 \\
\hline GO:0009791 & post-embryonic development & 578 \\
\hline GO:0000003 & reproduction & 541 \\
\hline GO:0006629 & lipid metabolic process & 517 \\
\hline GO:0007165 & signal transduction & 512 \\
\hline GO:0007049 & cell cycle & 498 \\
\hline GO:0009653 & anatomical structure morphogenesis & 498 \\
\hline GO:0009607 & response to biotic stimulus & 482 \\
\hline GO:0006412 & translation & 427 \\
\hline GO:0006519 & cellular amino acid metabolic process & 376 \\
\hline GO:0009719 & response to endogenous stimulus & 369 \\
\hline GO:0030154 & cell differentiation & 356 \\
\hline GO:0009908 & flower development & 329 \\
\hline GO:0009790 & embryo development & 302 \\
\hline GO:0006091 & generation of precursor metabolites and energy & 277 \\
\hline GO:0040029 & regulation of gene expression, epigenetic & 256 \\
\hline GO:0016049 & cell growth & 224 \\
\hline GO:0019748 & secondary metabolic process & 209 \\
\hline GO:0006355 & regulation of transcription, DNA-templated & 183 \\
\hline GO:0055114 & oxidation-reduction process & 180 \\
\hline GO:0006351 & transcription, DNA-templated & 170 \\
\hline GO:0006468 & protein phosphorylation & 149 \\
\hline GO:0006886 & intracellular protein transport & 118 \\
\hline GO:0009605 & response to external stimulus & 112 \\
\hline GO:0008219 & cell death & 109 \\
\hline GO:0055085 & transmembrane transport & 107 \\
\hline GO:0006457 & protein folding & 100 \\
\hline
\end{tabular}

at least 2 reads per million quantile normalized reads (rpmqn) in the higher expressed stage, and a two-fold expression change in the transition between the respective stages. The expression analysis resulted in $756 \mathrm{DE}$ transcripts for the first transition (T1) and 5,629 DE transcripts for T2 (Additional file 6: Table S6). In both transitions the majority of transcripts is downregulated, $66.67 \%$ in T1 and $56.96 \%$ in T2. 301 (39.81\%) of the DE transcripts after the cold-stress treatment in T1 exhibit also $\mathrm{DE}$ in T2. The proportion of the number of up- and downregulated transcripts in $\mathrm{T} 1$ resembles a previous microarray-based study for the effect of mannitoltreatment on microspore embryogenesis in barley [26].

The correlation-based cluster analysis of the expression stage specific expression values (Fig. 2c) suggested more differences in gene expression in T2 than in T1. These results were supported by a principal component analysis (PCA) for all DE transcripts in at least one stage transition, which resulted in a clear separation of the first two microspore stages S1 and S2 from the later stage S3, explaining $72.45 \%$ of the variance (Additional file 7: Figure S1). The similarity of S1 and S2 in comparison to S3 in the PCA highlights that this separation pattern is not a result from higher expression variation between the replicates that could have been potentially caused by the manual sampling of the microspores, but differential expression of specific sets of transcripts.

A k-means cluster analysis for all DE transcripts was performed to uncover expression switches throughout the two stage transitions (see Fig. 4). In agreement with the expression comparison (Fig. 2c) as well as with the results from the PCA the clustering resulted predominantly in two major expression pattern clusters, with basically either up (cluster 1, 9 and 12; see Fig. 4a, Fig. 4i and Fig. 4l) or down (cluster 3 and 5; see Fig. 4c and Fig. 4e) regulation of expression between the microspore stages S2 and S3. Another expression pattern is up-/ downregulation specifically after the stress treatment in T2 with reversion of the expression pattern towards T3 given for clusters 4, 6 and 7 (see Fig. 4d, Fig. 4f and Fig. 4g). Interestingly only clusters exhibiting a steady decrease (cluster 10 and 11; see Fig. 4j and Fig. 4k) but none for steady increase of gene expression could be observed. Changes in gene expression either up or down in T1 is given only for a smaller number of transcripts (cluster 2 and 8; see Fig. 4b and Fig. 4h).

The clusters were inspected for known regulatory transcripts, which signify the transition from the gametophytic to the embryonic developmental program. Strikingly, cluster 1 contains a transcript with homology to the embryogenesis related transcription factor $B A B Y B O O M 2$ (BBM2, transcript 4758). Interestingly, the major clusters 1 and 3 both contain various transcripts with homology to epigenetic 
Table 3 Overrepresented biological processes of transcript expression clusters

\begin{tabular}{|c|c|c|c|c|}
\hline Cluster & GO term & GO term description & Number of transcripts & Enrichment $p$-value \\
\hline 1 & GO:0006259 & DNA metabolic process & 90 & $<10^{-6}$ \\
\hline 1 & GO:0007049 & cell cycle & 78 & $<10^{-6}$ \\
\hline 1 & GO:0007018 & microtubule-based movement & 12 & $3 \cdot 10^{-6}$ \\
\hline 1 & GO:0007067 & mitotic nuclear division & 12 & $3 \cdot 10^{-6}$ \\
\hline 1 & GO:0040029 & regulation of gene expression, epigenetic & 44 & $1.1 \cdot 10^{-5}$ \\
\hline 1 & GO:0043531 & ADP binding & 16 & $1.6 \cdot 10^{-5}$ \\
\hline 1 & GO:0006275 & regulation of DNA replication & 12 & $7.9 \cdot 10^{-5}$ \\
\hline 1 & GO:0003677 & DNA binding & 111 & $9.6 \cdot 10^{-5}$ \\
\hline 1 & GO:0004803 & transposase activity & 8 & $1.86 \cdot 10^{-4}$ \\
\hline 1 & GO:0006260 & DNA replication & 15 & $2.86 \cdot 10^{-4}$ \\
\hline 1 & GO:0003774 & motor activity & 12 & $3.81 \cdot 10^{-4}$ \\
\hline 1 & GO:0006418 & tRNA aminoacylation for protein translation & 10 & $7.99 \cdot 10^{-4}$ \\
\hline 1 & GO:0008017 & microtubule binding & 6 & $9.88 \cdot 10^{-4}$ \\
\hline 1 & GO:0006313 & transposition, DNA-mediated & 7 & $1.01 \cdot 10^{-3}$ \\
\hline 1 & GO:0007131 & reciprocal meiotic recombination & 9 & $1.21 \cdot 10^{-3}$ \\
\hline 1 & GO:0000911 & cytokinesis by cell plate formation & 11 & $1.69 \cdot 10^{-3}$ \\
\hline 1 & GO:0006281 & DNA repair & 18 & $2.16 \cdot 10^{-3}$ \\
\hline 1 & GO:0010332 & response to gamma radiation & 7 & $2.95 \cdot 10^{-3}$ \\
\hline 1 & GO:0006261 & DNA-dependent DNA replication & 7 & $3.88 \cdot 10^{-3}$ \\
\hline 1 & GO:0009909 & regulation of flower development & 13 & $4.31 \cdot 10^{-3}$ \\
\hline 1 & GO:0003676 & intracellular protein transport & 77 & $5.49 \cdot 10^{-3}$ \\
\hline 1 & GO:0006886 & intercellular protein transport & 19 & $6.59 \cdot 10^{-3}$ \\
\hline 1 & GO:0016043 & cellular component organization & 140 & $8.09 \cdot 10^{-3}$ \\
\hline 5 & GO:0009987 & cellular process & 205 & $5.18 \cdot 10^{-4}$ \\
\hline 5 & GO:0019538 & protein metabolic process & 59 & $5.25 \cdot 10^{-4}$ \\
\hline 5 & GO:0051603 & proteolysis involved in cellular protein catabolic process & 5 & $1.3 \cdot 10^{-3}$ \\
\hline 5 & GO:0005839 & proteasome core complex & 5 & $1.75 \cdot 10^{-3}$ \\
\hline 5 & GO:0006810 & transport & 91 & $3.29 \cdot 10^{-3}$ \\
\hline 9 & GO:0009220 & pyrimidine ribonucleotide biosynthetic process & 7 & $1.1 \cdot 10^{-4}$ \\
\hline 9 & GO:0003735 & structural constituent of ribosome & 12 & $3.43 \cdot 10^{-4}$ \\
\hline 9 & GO:0006412 & translation & 25 & $3.16 \cdot 10^{-3}$ \\
\hline 9 & GO:0006094 & gluconeogenesis & 5 & $3.68 \cdot 10^{-3}$ \\
\hline 9 & GO:0009560 & embryo sac egg cell differentiation & 5 & $5.98 \cdot 10^{-3}$ \\
\hline 10 & GO:0009058 & biosynthetic process & 85 & $<10^{-6}$ \\
\hline 10 & GO:0006629 & lipid metabolic process & 42 & $<10^{-6}$ \\
\hline 10 & GO:0009987 & cellular process & 138 & $4.7 \cdot 10^{-5}$ \\
\hline 10 & GO:0009056 & catabolic process & 65 & $1.17 \cdot 10^{-4}$ \\
\hline 10 & GO:0019748 & secondary metabolic process & 19 & $1.37 \cdot 10^{-4}$ \\
\hline 10 & GO:0008152 & metabolic process & 62 & $2.1 \cdot 10^{-4}$ \\
\hline 10 & GO:0005975 & carbohydrate metabolic process & 44 & $5.72 \cdot 10^{-4}$ \\
\hline 10 & GO:0019538 & protein metabolic process & 39 & $1.17 \cdot 10^{-3}$ \\
\hline 10 & GO:0006091 & generation of precursor metabolites and energy & 19 & $3.9 \cdot 10^{-3}$ \\
\hline 10 & GO:0006519 & cellular amino acid metabolic process & 23 & $6.15 \cdot 10^{-3}$ \\
\hline 11 & GO:0009058 & biosynthetic process & 60 & $<10^{-6}$ \\
\hline
\end{tabular}


Table 3 Overrepresented biological processes of transcript expression clusters (Continued)

\begin{tabular}{|c|c|c|c|c|}
\hline 11 & GO:0008152 & metabolic process & 45 & $<10^{-6}$ \\
\hline 11 & GO:0006629 & lipid metabolic process & 35 & $<10^{-6}$ \\
\hline 11 & GO:0005975 & carbohydrate metabolic process & 35 & $<10^{-6}$ \\
\hline 11 & GO:0006091 & generation of precursor metabolites and energy & 21 & $<10^{-6}$ \\
\hline 11 & GO:0019748 & secondary metabolic process & 17 & $<10^{-6}$ \\
\hline 11 & GO:0009056 & catabolic process & 42 & $1.8 \cdot 10^{-5}$ \\
\hline 11 & GO:0005488 & binding & 51 & $1.09 \cdot 10^{-4}$ \\
\hline 11 & GO:0006096 & glycolytic process & 7 & $2 \cdot 10^{-4}$ \\
\hline 11 & GO:0009987 & cellular process & 77 & $2.75 \cdot 10^{-4}$ \\
\hline 11 & GO:0006519 & cellular amino acid metabolic process & 17 & $5.5 \cdot 10^{-4}$ \\
\hline 11 & GO:0015979 & photosynthesis & 7 & $6.02 \cdot 10^{-4}$ \\
\hline 11 & GO:0055114 & oxidation-reduction process & 10 & $1.86 \cdot 10^{-3}$ \\
\hline 11 & GO:0009628 & response to abiotic stimulus & 29 & $2.28 \cdot 10^{-3}$ \\
\hline 11 & GO:0006950 & response to stress & 39 & $2.8 \cdot 10^{-3}$ \\
\hline
\end{tabular}

components such as the Argonaute genes AGO4 (transcript_3992), AGO5 (transcript_1301) and AGO6 (transcript_2354), the dicer-like gene DCL3 (transcript_378), a large number of chromatin remodelling factors, such as various DNA-methyltransferases (DDM1, transcript_1568; DRM2, transcript_1831; $M E$ T1, transcript_3460; CMT3, transcript_6805), histone methyltransferases (NSD3, transcript_633; SUVH1, transcript_1921; SUVR5, transcript_1884) as well as the histone deacetylase (HD2A, transcript_5605) in cluster 1 . The opposing cluster 3 contains DCL1 (transcript_3100), the DNA-methyltransferase (DRM1, transcript_5286), as well as various histone deacetylases (HDA6, transcript_4537; HDA19, transcript_2818). The

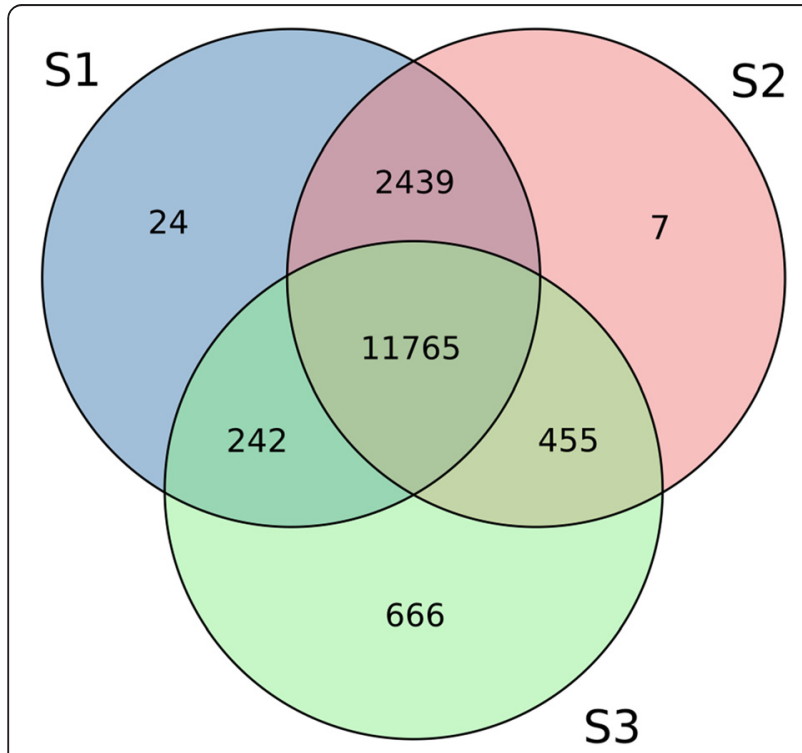

Fig. 3 Overlap of expressed transcripts in the three analyzed stages histone deacetylases HDA6 and HDA19 have been shown to be suppressors of embryonic properties [27] and thus were rightly found in cluster 3 . Likewise, changes in histone methylation and acetylation are associated with cell totipotency during microspore reprogramming to embryogenesis [9]. In agreement with other studies on androgenesis in various species [8-10] the large number of epigenetic components we found to be differentially expressed between the stages highlights their importance in the reprogramming of immature microspores to embryogenic cell fate.

Interestingly, homologues of previously discussed embryogenesis-marker genes are covered by the de novo assembled transcripts, such as SOMATIC EMBRYOGENESIS RELATED KINASE 1 (SERK1) [7] or LATE EMBRYO ABUNDANT (LEA) [28]. Unexpectedly, we found SERK1 with highest expression in fresh microspores and the expression level decreases through both transitions. This is in agreement with the finding that SERK1 was essential for male gametophyte production [29] and indicates that its expression pattern is not exclusively attributed to embryogenic reprogramming. We found $L E A$ to be expressed at low levels in all three stages without any significant changes in expression levels neither after induction-treatment (T1) nor towards induced embryogenesis (T2). Thus the transcription profiles of these known embryogenesis-marker genes do not indicate their involvement in the reprogramming of wheat microspores.

\section{GO enrichment analysis}

To further functionally characterize the stage transitions and expression clusters we performed a GO enrichment analysis for DE transcripts. The full results are listed in Additional file 8: Table S7 and Additional file 9: Table S8, 


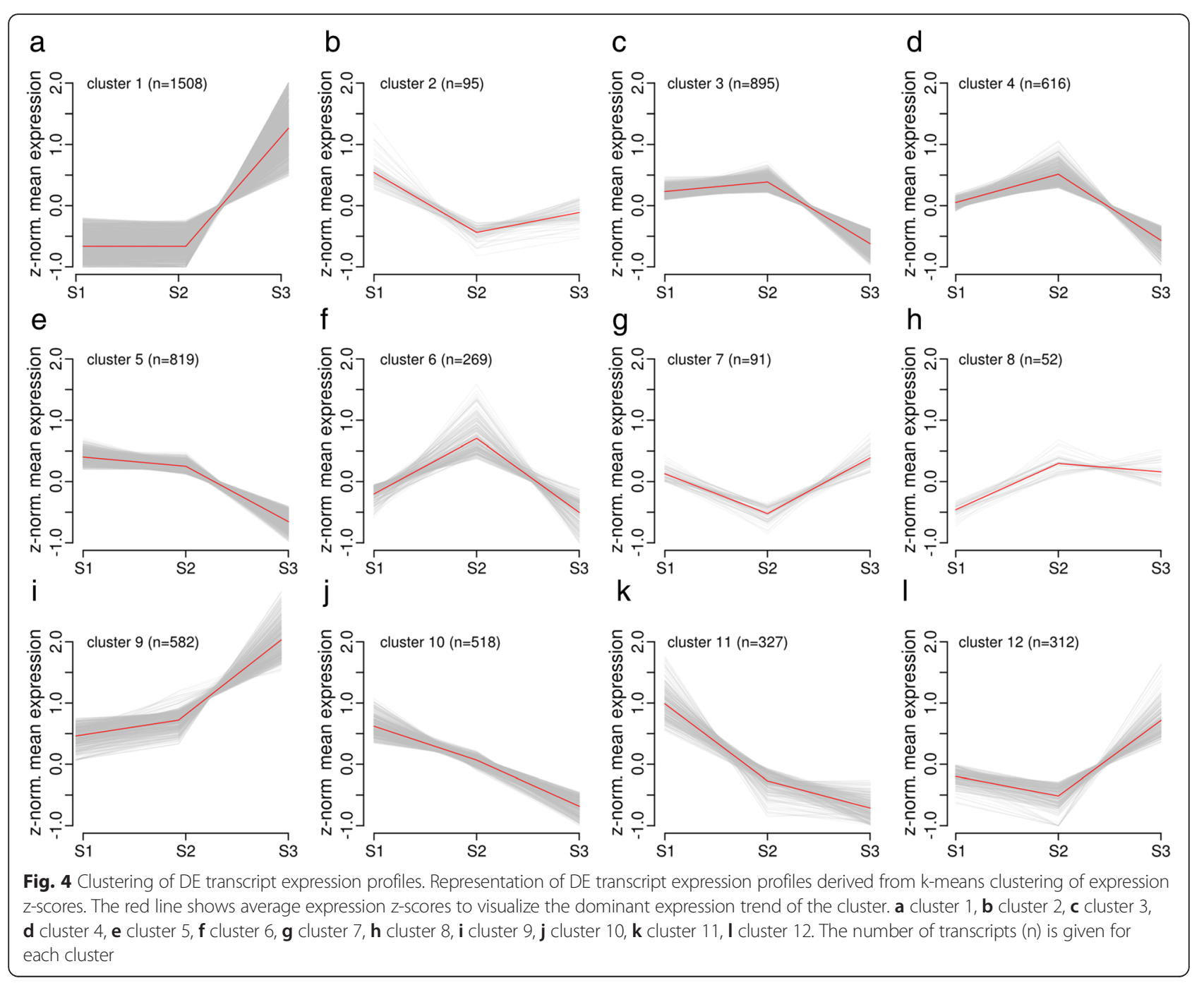

for the transitions and the expression pattern clusters, respectively. Additionally, major enrichments of the expression clusters are shown in Table 3. In T1, GO terms were only found to be enriched for downregulated transcripts, namely, amongst others, "carbohydrate metabolic process", "vacuole", and "response to stress", which all likely represent the dedifferentiation of the microspores due to the inductive treatment. A large set of GO terms overlaps among the downregulated transcripts in both transitions such as "generation of precursor metabolites" and "energy", "lipid metabolic process", "metabolic process", "catabolic process", "biosynthetic process", and "response to abiotic stimulus", which presumably represent sustained dedifferentiation from the microgametophytic pathway. The set of GO terms for upregulated transcripts in T2 contains the general terms protein binding, DNA binding and nucleic acid binding, most likely reflecting initiation of embryogenic transcription and protein machinery. Likewise, the GO terms "cell cycle", "cellular component organization" as well as numerous microtubule and mitosis related terms, "histone H3K9 methylation", "DNA methylation" and "histone phosphorylation" were enriched among upregulated transcripts in T2. The indicated downregulation of metabolic and biosynthetic processes in both transitions with concurrent upregulation of chromatin modifications and organization of cellular components as well as the cell cycle in T2 is in agreement with a cell cycle arrest, which was suggested to be required for the reprogramming to embryogenic fate before the cell cycle is again released [1]. The GO term "H3K9 methylation" for upregulated transcripts in T2 is in accord with the finding of increased H3K9 methylation in embryo-like structures as compared to microspores [9].

Cluster 1 exhibits an enrichment for various GO-terms reflecting karyokinesis, the microspores are undergoing in T2, such as "microtubule binding", "cytokinesis by cell plate formation", "DNA-dependent DNA replication" and "cytoskeleton". The stress-induced rearrangement of the cytoskeleton followed by a symmetric division of the microspore has been described in various studies as initial 
steps towards microspore embryogenesis (see review [2]). Although cluster 3 is the second largest cluster, it is only enriched for the single GO-term "endoplasmic reticulum". That there are no other terms enriched, might reflect that the downregulation of transcript expression in T2 covers a multitude of functions and processes. In contrast, cluster 5 with a similar expression pattern but continuous downregulation in $\mathrm{T} 1$ and $\mathrm{T} 2$ has an enrichment for numerous GO-terms for protein related processes, such as "protein metabolic process", "proteolysis involved in cellular processes", "Golgi apparatus" and "proteasome core complex", which might reflect the previously described degradation of gametophytic cell fate-related proteins to allow for a reprogramming towards embryogenesis. Especially, the enrichment for "Golgi apparatus" might resemble findings in Brassica napus where autophagy and cytoplasmic cleaning by excretion was found to be unique to microspores undergoing reprogramming to an embryogenic fate: In contrast to non-responding microspores, freshly isolated microspores at the vacuolated stage, which were optimal for induction, exhibit Golgi-stacks [30]. Surprisingly, the corresponding genes show equal expression levels in untreated isolated microspores and after the stress-treatment. This might indicate an early transcriptional stress response to the mannitol buffer and would fit to a similar observation by Marashin et al. [3]. Cluster 9 is enriched for GO-terms related to transcription and translation: "structural constituent of ribosome", "ribosome", "DNA binding", "translation" and "DNA-directed RNA polymerase activity" and is likely related to the establishment of an embryogenic program. Interestingly, cluster 9 exhibits also enrichment for the GO-term "embryo sac egg cell differentiation", which might be indicative for the reprogramming of the microgametophytic pathway. Although cluster 10 and 11 represent progressive downregulation of transcripts and both cover only a relative small amount of DE transcripts, they exhibit enrichments for a large number of GO-terms related to catalytic activity and various metabolic processes, which might relate to downregulation of microgametophytic pathways. The additional enrichment for various stress-related terms, such as "oxidation-reduction process", "response to abiotic stimulus", "response to stress" and interestingly "embryo development" in cluster 11 was unexpected, since it has been shown, that the anther pre-treatment activates plant defense gene expression in response to mannitol solution and cold stress treatment [31]. Considering the decreasing expression levels with initiated embryogenesis the latter GO term most likely represents suppressors of embryo development.

\section{sRNA sequencing results}

The sRNA-seq resulted in 92.54 Mio. clean reads, with 9.71 Mio. to 10.79 Mio. reads per library (see Table 1).
In total 19.63 Mio. distinct sequences were obtained, with 1.8 Mio. to 4 Mio. distinct sequences per library (see Table 1). The sRNA length distribution exhibits a peak at 24-nt for all replicates of all three stages, representing the most abundant short interfering RNAs (siRNA). However, two of the three replicates from S1 showed a smaller fraction of 24-nt sRNAs (Fig. 5a). The length distribution of distinct sRNA reads exhibited an additional peak for 21-nt sRNAs (Fig. 5b), a fraction of which most likely represents microRNAs (miRNA). The fraction of 24-nt sRNAs exhibited a higher variability than given for the total sRNA length distribution in contrast to the sRNA lengths from 15-nt to 20-nt as well as 25-nt to 28-nt, which, except of 20-nt sRNAs, are not representing known functionally active sRNA classes [32].

\section{sRNA expression analysis}

Distinct sRNAs were defined as expressed if their expression was equal or higher than $1 \mathrm{rpmqn}$, this criterion was satisfied for 63,880 to 70,478 sRNAs per library. A comparison of expression values between all replicates of the three stages revealed a high similarity for the three replicates of each stage (Fig. 5c), again reflecting the overall uniformity of the biological replicates beside the differences in abundance of RNAs of specific length. The variance between replicates for sRNAs is higher than for transcripts. A possible explanation provides the less specific generation of siRNAs from various genomic loci in contrast to the defined gene loci of transcripts. In contrast to the transcript expression, the correlation between the replicates revealed drastic expression changes from S1 to S2 as well as S2 to S3, as the replicates of S2 are less correlated to S1 and S3 than S1 and S3 to each other. This drastic difference to transcript expression pattern might be explainable by stress-induced activation of transposons resulting in the generation of new sets of siRNAs and delayed effects on gene expression by de novo methylation of target TEs [33, 34]. Furthermore this difference might be attributed to the different isolation procedures of microspores for mRNA and sRNA sequencing: In contrast to individual selection of microspores with specific morphology for mRNA sequencing (Fig. 1), the gradient centrifugation, we used to isolate microspores for sRNA sequencing, enrich for living microspores only and thus more likely include sRNAs from microspores undergoing cell fates other than embryogenesis.

DE sRNAs were determined from all sRNAs with an expression level of at least 2 rpmqn in the higher expressed stage, a minimum of two-fold expression change. The expression analysis with these thresholds resulted in 867 DE sRNAs for T1, with 830 (95.73\%) being upregulated and 37 (4.27 \%) being downregulated 


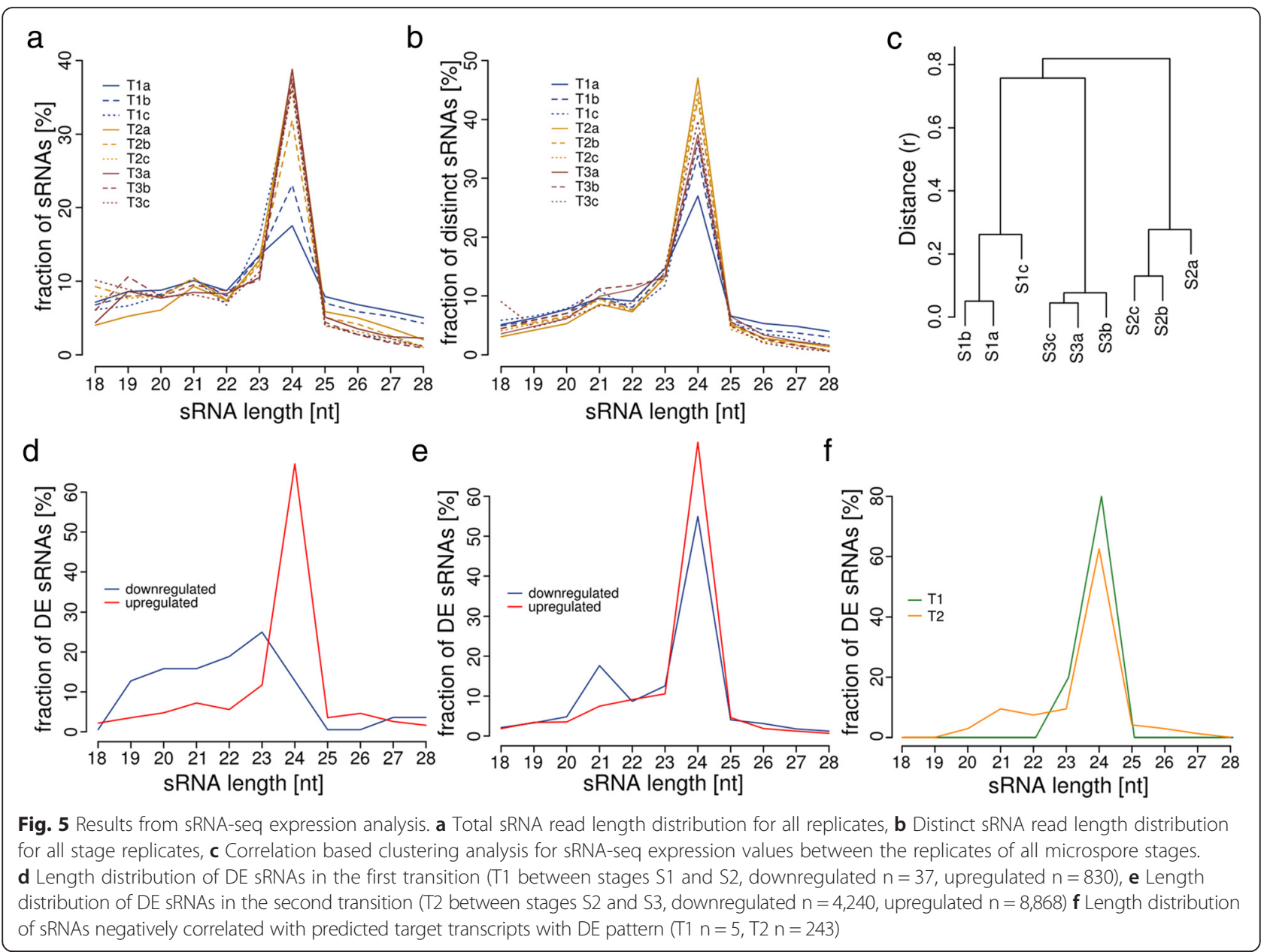

(Additional file 10: Table S9, Additional file 11: Table S10). The upregulated sRNAs account primarily to 24-nt sRNAs while the downregulated sRNAs are scattered from 19-nt to 23-nt (Fig. 5d). For T2 13,108 DE sRNAs were identified in total, with 8,868 (67.65 \%) being upregulated and 4,240 (32.35 \%) being downregulated (Additional file 10: Table S9, Additional file 11: Table S10). In T2, 24-nt sRNAs accounted for the majority of up as well as downregulated sRNAs. Furthermore, the downregulated sRNAs exhibited a high fraction of 21-nt sRNAs (Fig. 5e). 304 of the DE sRNAs overlap between T1 and T2 that all are of 24-nt length.

In the various developmental stages analyzed, 66 of 119 known mature miRNAs of wheat listed in miRBase release 21 [35] were found to be expressed. Three of these (taemiR9669-5p, tae-miR397-5p, and tae-miR9658-3p) showed upregulation, whereas one (tae-miR9672b) showed downregulation in T2. Consistent with a putative role in microspore embryogenesis, which involve the generation of undifferentiated multicellular structures at first, miR397 was shown to be highly expressed in undifferentiated but not in differentiated rice embryogenic calli from somatic tissues [36]. Interestingly, miR397 is upregulated under cold conditions [37] and overexpression resulted in higher cold stress tolerance in Arabidopsis [38]. In wheat microspores, cold inducibility of miR397 might be reduced or delayed, since we revealed no upregulation in S2 right after the cold stress treatment but in the later stage S3. Another miRNA, which might be involved in the regulation of androgenesis, is tae-miR9658, since it was shown to be highly expressed in developing grains but less abundant in vegetative tissues [39].

\section{Prediction of sRNA target transcripts}

To identify potential regulatory effects of sRNAs on mRNAs we predicted sRNA targets among the assembled transcripts for all DE sRNAs. The target prediction resulted in 97 putative target transcripts for DE sRNAs in T1 and 1,179 putative sRNA target transcripts in T2. Five sRNA/target pairings in T1 exhibited DE transcripts and a strong negative correlation between sRNA and target expression. All these targets were downregulated from S1 to S2. For T2, we found 251 DE target transcripts of which 133 exhibit a strong negative correlation 
with the targeting sRNA expression values, 86 of these targets were up and 47 were downregulated from S2 to S3 (Additional file 12: Table S11). No DE targets could be identified among the assembled contigs for any of the 4. DE known miRNAs listed in miRBase release 21 [35].

Although we used different microspore isolation procedures for mRNA and sRNA sequencing, both datasets are highly related by identical tissue culture conditions and staging. Thus, a negative correlation between sRNA and targeted transcript expression should support the involvement of the sRNA in regulation of the target transcript either by post-transcriptional gene silencing (PTGS) or transcriptional gene silencing (TGS) [40]. The targeted transcripts identified include a number of genes indicated to be involved in embryogenesis or even androgenesis, such as HDA19 (transcript_2818), as already discussed in the context of the $\mathrm{DE}$ transcripts analysis, which is downregulated in T2. HDA19 has been shown to contribute to the repression of embryogenesisrelated genes after germination [27]. It has been furthermore shown that histone deacetylase inhibitors promote totipotency to microspores [41]. Chromatin modifications are suggested to be required for the accessibility of embryogenesis-related genes [9]. Another interesting gene is PROLIFERA (PRL), represented by transcript_9383, which is upregulated in T2. PRL has been shown to be involved in megaspore and embryo development but not in the developing microgametophyte [42] and thus might represent the deviation from the gametophytic cell fate. TaCer1 (transcript_178), which is highly increased in microspores after stress treatment (S2) and is 528-fold downregulated in T2, represents ECERIFERUM1 a putative decarbonylase involved in cuticular wax biosynthesis from very long chain fatty acids (VLCFA), which can be regulated by various stresses [43]. It was suggested that Cer1 is involved in stress tolerance by modulating the aldehydes/alkanes ratio of cuticular wax in wheat as well as other plants [43-46]. A previous study suggested the involvement of wax biosynthesis from VLCFA in wheat microspore embryogenesis as a pathway resulting in signal molecules leading to controlled cell division in absence of surrounding tissue [47]. Overall, only 48 of $666 \mathrm{DE}$ target genes potentially regulated by sRNAs obtained an annotation. We expect the remaining set of transcripts to contain important regulatory genes involved in the alteration of cell fate towards embryogenic development, not yet discovered by other studies.

\section{Prediction of miRNA precursors}

To differentiate between potential PTGS and TGS, miRNA precursors on wheat genomic sequences were predicted. The prediction of miRNA precursors (pre-miRNA) revealed in total 1,717 distinct candidates (Additional file 13:
Table S12). 39 pre-miRNAs candidates showed homology to known pre-miRNAs (Table 4). The comparison of the predicted mature miRNAs with the identified sRNA/target pairs revealed two putative miRNAs, namely sRNA 1167446 potentially targeting transcript_1777 with high homology to the Aegilops tauschii predicted protein F775_10365 of unknown function and sRNA 6202839 targeting transcript_5689 with high homology to the wheat cDNA TRAES_3AS_647411E39.2 of unknown function. These results with nearly no consensus between predicted mature miRNAs and the sRNAs with predicted targets suggests that miRNA-mediated post-transcriptional regulation does not explain the majority of the predicted sRNA/ target pairs with high negative correlation. In the light of the high number of chromatin modifiers identified to be expressed, it is assumed that different sRNA-guided TGS mechanisms such as chromatin modifications and DNA methylation are involved in the induction of microspore embryogenesis in wheat. This assumption is supported by the high variability of the number of distinct 24-nt sRNAs between stages (Fig. 5b), as well as the high fraction of DE 24-nt sRNAs negatively correlated with their potential target transcript expression (Fig. 5f), as 24-nt sRNAs were shown to be predominantly involved in TGS [32].

\section{Conclusions}

Our study provides the first large-scale transcriptome dataset for microspore embryogenesis induction in wheat. The de novo assembly and mapping to public wheat cDNA sequences resulted in a high number of novel transcripts. A major part of these might be presumably largely specific transcripts for microspore embryogenesis induction. A GO annotation revealed a large fraction of stress as well as embryo development related transcripts. Many transcripts were found to be specifically expressed in microspores undergoing their first visible nuclear division, numerous with functions in embryogenesis or epigenetic mechanisms. While the inductive treatment resulted in $756 \mathrm{DE}$ transcripts, the following transition resulting in first nuclear divisions exhibits a larger set of 5,269 DE transcripts. A GO enrichment analysis for the DE transcripts revealed metabolism and biosynthesis related transcripts to be downregulated in both stage transitions whereas chromatin related transcripts were found to be enriched and thus represent a dedifferentiation of the developing microspore followed by a reprogramming towards embryogenic development. The sRNA sequencing mirrors the transcriptome in terms of numbers with 867 DE sRNAs in the first and 13,108 DE sRNAs in the second stage transition. Prediction of sRNA targets identified a large number of putative target transcripts, which contained genes previously shown to be involved in microspore or zygotic embryogenesis. Our results suggest epigenetic mechanisms related to TGS 
Table 4 Numbers of pre-miRNA candidates with homology to known pre-miRNAs in miRBase release 21 [35]

\begin{tabular}{|c|c|c|}
\hline Predicted pre-miRNA & $\begin{array}{l}\text { BLASTn top hit in } \\
\text { miRBase release } 21\end{array}$ & Organism \\
\hline miRCandidate1467 & osa-MIR159e & Oryza sativa \\
\hline miRCandidate1 & ata-MIR166a & Aegilops tauschii \\
\hline miRCandidate1079 & ata-MIR166b & Aegilops tauschii \\
\hline miRCandidate770 & ata-MIR166e & Aegilops tauschii \\
\hline miRCandidate1062 & bdi-MIR166g & $\begin{array}{l}\text { Brachipodium } \\
\text { distachion }\end{array}$ \\
\hline miRCandidate923 & bdi-MIR166g & $\begin{array}{l}\text { Brachipodium } \\
\text { distachion }\end{array}$ \\
\hline miRCandidate1425 & osa-MIR168a & Oryza sativa \\
\hline miRCandidate437 & ata-MIR393 & Aegilops tauschii \\
\hline miRCandidate1319 & osa-MIR396c & Oryza sativa \\
\hline miRCandidate675 & ata-MIR398f & Aegilops tauschii \\
\hline miRCandidate90 & tae-MIR1122a & Triticum aestivum \\
\hline miRCandidate 1040 & tae-MIR1 128 & Triticum aestivum \\
\hline miRCandidate1068 & tae-MIR1128 & Triticum aestivum \\
\hline miRCandidate 1370 & tae-MIR1128 & Triticum aestivum \\
\hline miRCandidate 30 & tae-MIR1128 & Triticum aestivum \\
\hline miRCandidate342 & tae-MIR1128 & Triticum aestivum \\
\hline miRCandidate45 & tae-MIR1128 & Triticum aestivum \\
\hline miRCandidate941 & tae-MIR1 128 & Triticum aestivum \\
\hline miRCandidate1469 & tae-MIR1135 & Triticum aestivum \\
\hline miRCandidate1585 & tae-MIR1135 & Triticum aestivum \\
\hline miRCandidate471 & tae-MIR1135 & Triticum aestivum \\
\hline miRCandidate720 & tae-MIR1135 & Triticum aestivum \\
\hline miRCandidate1023 & tae-MIR1136 & Triticum aestivum \\
\hline miRCandidate1678 & tae-MIR1136 & Triticum aestivum \\
\hline miRCandidate1641 & tae-MIR5048 & Triticum aestivum \\
\hline miRCandidate 1033 & tae-MIR5084 & Triticum aestivum \\
\hline miRCandidate 1332 & tae-MIR5084 & Triticum aestivum \\
\hline miRCandidate171 & tae-MIR9653a & Triticum aestivum \\
\hline miRCandidate166 & tae-MIR9653b & Triticum aestivum \\
\hline miRCandidate177 & tae-MIR9653b & Triticum aestivum \\
\hline miRCandidate823 & tae-MIR9657c & Triticum aestivum \\
\hline miRCandidate689 & tae-MIR9661 & Triticum aestivum \\
\hline miRCandidate309 & tae-MIR9671 & Triticum aestivum \\
\hline miRCandidate 1069 & tae-MIR9672a & Triticum aestivum \\
\hline miRCandidate1146 & ata-MIR9674a & Aegilops tauschii \\
\hline $\begin{array}{l}\text { miRCandidate883, } \\
\text { miRCandidate892 }\end{array}$ & tae-MIR9674a & Triticum aestivum \\
\hline miRCandidate930 & ata-MIR9674a & Aegilops tauschii \\
\hline miRCandidate884 & tae-MIR9674b & Triticum aestivum \\
\hline miRCandidate1355 & tae-MIR9777 & Triticum aestivum \\
\hline
\end{tabular}

rather than miRNA-based PTGS to be largely involved in the reprogramming of the microspore developmental fate. These results and the generated sequencing resources will contribute to a deeper understanding of the molecular mechanisms involved in the induction of wheat microspore embryogenesis.

\section{Methods}

\section{Plant material}

The Bulgarian winter wheat cultivar "Svilena", European Wheat Database accession: BG 2001-TRT-AE-125 [48], which is highly responsive to stress-induced microspore embryogenesis [12] was used for this study. Seeds of this cultivar were initially provided to us by the Institute of Wheat and Sunflower "Dobrudja", General Toshevo, Bulgaria and propagated by us thereafter. Donor plants were grown as described in Rubtsova et al. 2013 [13]. The inductive pre-treatment of spikes for 10 days and the following in vitro culture of anthers was performed as described in Rubtsova et al. 2013 [13].

\section{Microspore isolation}

Two different methods were used for microspore isolations: a) manual sampling for small sample sizes and b) purification of microspores using gradient centrifugation for bigger sample sizes. Three stages of microspore embryogenesis were isolated by both methods.

For transcriptome analysis, microspores were manually sampled under sterile conditions using a 10x objective (100x magnification) and an Eppendorf CellTram vario (Eppendorf AG, Hamburg, Germany). The first stage (S1) represented untreated microspores at the late vacuolated uninucleate stage and were isolated from freshly harvested spikes. At the second time point (S2), slightly enlarged microspores with a star-like structure were sampled directly after a 10-days cold pre-treatment of the spikes. For the third stage (S3), enlarged microspores showing first visible karyokinesis were isolated from invitro anther culture. Karyokinesis was generally visible after 4-8 days in culture. During this time, the developmental stages of the microspores were checked daily. When 5-10\% of microspores showed visible karyokineses, all anthers of one petri dish were used for isolation. Extensive preliminary tests were performed, which yielded in the isolation of only ten microspores from 2-8 anthers at a time to keep isolation time short. Microspores were isolated in $0.4 \mathrm{M}$ mannitol, directly frozen in $10 \mu \mathrm{l} 0.4 \mathrm{M}$ mannitol with liquid nitrogen, and stored at $-70{ }^{\circ} \mathrm{C}$ until further processing.

For sRNA analysis, the same in vitro culture conditions were applied and a gradient isolation of microspores was performed, which enriched for viable microspores but do not select for specific morphology. Also here, extensive preliminary tests were performed to 
optimize the concentration and purity of the individual developmental microspore stages S1 - S3. Since the amount of responding microspores is reduced with each stage, the number of spikes had to be adapted, e.g. for S3 up to 24 spikes were used for one isolation. For optimized microspore isolation, anthers were collected in $0.4 \mathrm{M}$ mannitol, homogenized, sieved and centrifuged at $98 \mathrm{~g}$ for $5 \mathrm{~min}$ to pellet the microspores. The precipitate was concentrated and washed 2 times in $0.4 \mathrm{M}$ mannitol at $98 \mathrm{~g}$ for $5 \mathrm{~min}$. The resuspension was stacked on $20 \%$ maltose and centrifuged at $98 \mathrm{~g}$ for $4 \mathrm{~min}$. During this step, all cell debris and non-viable microspores were discarded. The interphase was washed with $0.4 \mathrm{M}$ mannitol and centrifuged at $98 \mathrm{~g}$ for $10 \mathrm{~min}$ before resuspending in a small amount of $0.4 \mathrm{M}$ mannitol. The purity of each isolation was checked by light microscopy and the concentration of microspores was determined by counting microspores in $1 \mu \mathrm{l}$ suspension in triplicate.

\section{RNA preparation and sequencing}

For RNA-seq and sRNA-seq, 3 biological replicates for each of the 3 stages were used. For RNA-seq, mRNA was isolated from 100 hand-sorted microspores and cDNA libraries were generated according to the protocol described in Lê et al. 2005 [49]. RNA-seq library preparation of the sample replicates indexed with unique nucleic acid identifiers was performed using Illumina Nextera DNA Library Kit (Illumina Inc, San Diego, CA, USA). For sRNA-seq, total RNA was isolated using the mirVana miRNA Isolation Kit (Life Technologies Corp., Carlsbad, CA, USA) from $\sim 100,000$ gradient-sorted microspores. The quality of the RNA samples was tested and verified by BGI Tech Solutions Co., Ltd. (Hong Kong) on a 2100 Bioanalyzer (Agilent, Santa Clara, CA, USA) prior to sequencing library generation with the TrueSeq Small RNA Library Preparation Kit. Illumina sequencing of $50 \mathrm{bp}$ single-end reads was performed on Illumina Hi-Seq 2000 instruments (Illumina Inc, San Diego, CA, USA) by BGI Tech Solutions Co., Ltd. (Hong Kong).

\section{Sequencing data processing}

The RNA-seq data was generated with CASAVA 1.8.1 (Illumina Inc, San Diego, CA, USA) and initially processed by BGI Tech Solutions Co., Ltd. (Hong Kong) to remove adapter sequences, contamination and lowquality reads. The preprocessed RNA-seq reads were quality-trimmed to $99.9 \%$ sequencing quality using a custom Java program using the Picard API [50]. Trimmed reads with less than 40 bp were discarded. The sRNA-seq reads were trimmed from adapter sequences and quality-trimmed to $99.9 \%$ sequencing quality. All sRNA reads longer than 15 nt were retained for further analysis.

\section{Transcriptome de novo assembly and annotation}

All trimmed RNA-seq reads from all replicates of all three stages were merged and used for de novo assembly of a wheat microspore embryogenesis transcriptome. The assembly was performed using Trinity version r2013-08-14 [16] with default parameters.

The contigs were aligned to the NCBI non-redundant protein (nr) database (downloaded 12/13/2013, ftp.ncbi .nlm.nih.gov/blast/db) using BLASTx (version 2.2.29+) [51] with maximum e-value of $10^{-10}$ and a limitation to the 20 most significant alignments. Based on this mapping the contigs were GO annotated using Blast2GO [18] and Trinotate [19].

The assembled contigs were mapped to wheat cDNA sequences from ensembl release 26 [20] using BLASTn (version 2.2.29+) [51] to identify fragmented assemblies. Multiple contigs mapping to one cDNA were clustered to a single transcript identifier. GO terms obtained for transcripts with fragmented assembly were merged.

\section{sRNA annotation}

The sRNA sequences were mapped to mature miRNAs listed for wheat in miRBase release 21 [35] using the sequence aligner Bowtie (version 1.01) [52] without mismatches.

\section{Expression analysis}

The expression patterns of the transcripts identified by the de novo assembly were calculated by counting all reads uniquely mapping to the contigs of transcript clusters using the sequence aligner Bowtie (version 1.01) [52] allowing one mismatch. The sample replicates were hierarchically clustered based on their Pearson correlation coefficients.

The sRNA sequences were collapsed to unique sequence reads and read counts were determined.

Read count data from transcriptome as well as sRNA were individually normalized by quantiles normalization [53] with a modification preventing the allocation of expression values to transcripts not expressed in the sample. The quantile normalized expression values were finally scaled to 1 Mio. quantiles normalized reads (rpmqn). The sample replicates were hierarchically clustered based on their Pearson correlation coefficients with the complete linkage method in R [54].

\section{Differential expression analysis}

The DE of transcripts and analogously of sRNAs was tested individually for both transitions (T1 from stage S1 to S2, T2 from S2 to S3) for all transcripts or sRNAs, respectively, with at least two-fold expression change between the stages a minimal expression of 1 rpmqn in the higher expressed stage and a maximal standard deviation for the stage replicates of $25 \%$ from the average 
expression of the stage. The significance of DE was tested by Student's t-tests with a FDR of $5 \%$ [55] in a custom Java program using the Java Statistical Classes API [56].

\section{Cluster analysis of differentially expressed transcripts}

A grouping of the replicates of all microspore stages was performed by principal component analysis in $R$ [54] based on z-normalized expression data for all transcripts as well as separately for all DE transcripts in at least one transition.

A k-means cluster analysis of mean z-normalized stage replicate transcript expression means with $\mathrm{k}$ set to 12 clusters was performed in Gene Cluster 3.0 using the centered correlation as dissimilarity measure [57].

\section{GO enrichment analysis for transcript expression pattern clusters}

The enrichment for GO terms was tested for all clusters in a bootstrap analysis with 1 Mio. runs in a custom Java program, all terms with at least 5 transcripts and a FDR [55] below $5 \%$ were considered to be enriched.

\section{SRNA target prediction}

A sRNA target prediction was performed for all DE sRNAs on the de novo assembled contigs from the RNA-seq using psRNATarget with default parameters [58]. All putative sRNA/target pairs were subsequently filtered by correlation analysis of the sRNA and transcript expression. Only sRNA/target pairs with strong negative correlation were retained as putatively sRNA regulated transcripts.

\section{miRNA prediction}

A prediction of miRNA precursors for the identification of miRNAs was performed using miRDeep-P (version 1.3) [59] individually for all stage replicates using the IWGSP1 chromosome based draft sequence [60] as genome. The pre-miRNAs were searched for homology to known pre-miRNAs in miRBase release 21 [35] using BLASTn (version 2.2.29+) [51]. The predicted mature miRNAs were filtered for sRNAs with predicted targets from the sRNA target prediction.

\section{Ethics approval and consent to participate}

Our experimental research complied with institutional, national, or international guidelines. We did not use any endangered species and complied with the Convention on the Trade in Endangered Species of Wild Fauna and Flora.

\section{Consent to publish}

Not applicable

\section{Availability of data and materials}

The data sets supporting the results of this article are available in the NCBI BioProject repository, under accession PRJNA297977, [http://www.ncbi.nlm.nih.gov/bio project/PRJNA297977]. This Transcriptome Shotgun Assembly project has been deposited at DDBJ/ENA/GenBank under the accession GDTJ00000000. The version described in this paper is the first version, GDTJ01000000.

\section{Additional files}

Additional file 1: Table S1. Transcript reconstruction. Reconstruction of de novo assembled contigs to wheat transcripts based on known wheat cDNAs. (XLS 1968 kb)

Additional file 2: Table S2. BLASTx hits of transcripts. BLASTx top hit results for de novo assembled contigs of restructured transcripts. (XLS $4120 \mathrm{~kb}$ )

Additional file 3: Table S3. GO annotation for transcript reconstruction. GO annotation for restructured de novo assembled transcripts based on functional homology using Blast2GO [16] and Trinotate [17]. (XLS $1212 \mathrm{~kb}$ )

Additional file 4: Table S4. GO annotation counts for the assembled transcripts. Summary of retrieved GO annotations for de novo assembled, restructured transcripts. (XLS 263 kb)

Additional file 5: Table S5. Stage specific transcripts. Listing of restructured de novo assembled transcripts with stage-specific expression (at least 0.5 qnrpm in all replicates) including BLASTx top hits. (XLS 88 kb)

Additional file 6: Table S6. DE transcripts in T1 and T2. Listing of transcript identifiers for DE, restructured de novo transcripts up- and downregulated in the stage transitions. (XLS $275 \mathrm{~kb}$ )

Additional file 7: Figure S1. PCA results for DE transcripts. PCA plot for DE transcript expression patterns resulting in clear separation of sample stage replicates. (PNG $55 \mathrm{~kb}$ )

Additional file 8: Table S7. Enriched GO terms for DE transcripts in the stage transitions. Listing of GO terms enriched for DE transcripts in stage transitions seperately for up- and downregulated expression patterns with term description, number of transcripts, and p-value. (XLS 35 kb)

Additional file 9: Table S8. Enriched GO terms for DE transcripts clustered by expression patterns. Listing of $\mathrm{GO}$ terms enriched for k-means clustered expression patterns of DE transcripts with description, number transcripts, and p-value. (XLS $27 \mathrm{~kb}$ )

Additional file 10: Table S9. DE sRNAs in T1 and T2. Listing of identifiers of DE sRNA up- and downregulated in the stage transitions. (XLS 349 kb)

Additional file 11: Table S10. Sequences of DE sRNAs and predicted miRNAs. Listing of all DE sRNAs as well as miRNAs with their sRNA identifier (XLS 1022 kb)

Additional file 12: Table S11. Putative sRNA/target pairs identified from negatively correlated DE sRNAs and DE transcripts. Listing of sRNA/ target pairs based on negative correlation of DE sRNA and DE transcript expression patterns that were identified using psRNATarget [58]. Stage transitions of DE, correlation coefficient, and transcript BLASTx top hits are listed. (XLS $36 \mathrm{~kb}$ )

Additional file 13: Table S12. miRNA prediction results. Results from miRNA precursor predictions using miRDeep-P [59] performed from sRNA sequencing data individually for all stage replicates. (XLS $790 \mathrm{~kb}$ )

\section{Abbreviations}

CDNA: complementary RNA; DE: differentially expressed; FDR: false discovery rate; GO: gene ontology; mRNA: messenger RNA; miRNA: microRNA; NCBI: National Center for Biotechnology Information; PCA: principal component analysis; pre-miRNA: miRNA precursor; PTGS: post-transcriptional gene silencing; RNA-seq: transcriptome sequencing; rpmqn: reads per million quantile normalized reads; 
sRNA: small RNA; sRNA-seq: small RNA sequencing; siRNA: small interfering RNA; TGS: transcriptional gene silencing; VLCFA: very long chain fatty acids.

\section{Competing interest}

The authors declare that they have no competing interests.

\section{Authors' contributions}

FS analyzed and interpreted the data and wrote the manuscript. SB performed the microspore isolation. JK introduced the isolation technique of individual microspores and provided the facilities. HG participated in the design of the study, conducted the microspore isolation and helped writing the paper. SS designed the study, conducted the sequencing experiments, interpreted the data and wrote the manuscript. All authors read and approved the final manuscript.

\section{Acknowledgements}

The authors thank Dominika Rybka for excellent technical assistance with RNA isolation from limited sample material.

\section{Funding}

This work was funded by the German ministry for education and research (BMBF, grant "SPEEDWHEAT" 031A108A-C). The funding body had no role in design of the study, analysis, and interpretation of data and in writing the manuscript.

\section{Author details}

${ }^{1}$ Developmental Biology, Biocenter Klein Flottbek, University of Hamburg, Ohnhorststrasse 18, 22609 Hamburg, Germany. ${ }^{2}$ Saaten-Union Biotec GmbH, Am Schwabenplan 6, 06466 Seeland, OT Gatersleben, Germany. ${ }^{3}$ Plant Reproductive Biology, Leibnitz Institute of Plant Genetics and Crop Plant Research (IPK), 06466 Seeland, OT Gatersleben, Germany. ${ }^{4}$ Institute for Plant Breeding, Seed Science and Population Genetics, University of Hohenheim, 70599 Stuttgart, Germany.

Received: 17 December 2015 Accepted: 14 April 2016

Published online: 21 April 2016

\section{References}

1. Seguí-Simarro JM, Nuez F. How microspores transform into haploid embryos: changes associated with embryogenesis induction and microspore-derived embryogenesis. Physiol Plant. 2008;134:1-12.

2. Soriano M, Li H, Boutilier K. Microspore embryogenesis: Establishment of embryo identity and pattern in culture. Plant Reprod. 2013;26:181-96.

3. Germanà MA. Gametic embryogenesis and haploid technology as valuable support to plant breeding. Plant Cell Rep. 2011;30:839-57.

4. Food and Agriculture Organisation of the United Nations. 2011. http://faostat.fao.org/default.aspx?lang5en. Accessed 14 March 2016

5. Maraschin SF, Caspers M, Potokina E, Wulfert F, Graner A, Spaink HP, et al. CDNA array analysis of stress-induced gene expression in barley androgenesis. Physiol Plant. 2006;127:535-50

6. Muñoz-Amatriaín M, Svensson JT, Castillo AM, Close TJ, Vallés MP. Microspore embryogenesis: assignment of genes to embryo formation and green vs. albino plant production. Funct Integr Genomics. 2009;9:311-23.

7. Maraschin SF. Androgenic switch: an example of plant embryogenesis from the male gametophyte perspective. J Exp Bot. 2005;56:1711-26.

8. El-Tantawy AA, Solís M, Risueño MC. Changes in DNA methylation levels and nuclear distribution patterns after microspore reprogramming to embryogenesis in barley. Cytogenet Genome Res. 2014;143:200-8.

9. Rodríguez-Sanz H, Moreno-Romero J, Solís MT, Köhler C, Risueño MC, Testillano PS. Changes in Histone Methylation and Acetylation during Microspore Reprogramming to Embryogenesis Occur Concomitantly with Bn HKMT and Bn HAT Expression and Are Associated with Cell Totipotency, Proliferation, and Differentiation in Brassica napus. Cytogenet Genome Res. 2014;143:209-18.

10. Solís M-T, El-Tantawy AA, Cano V, Risueño MC, Testillano PS. 5-azacytidine promotes microspore embryogenesis initiation by decreasing global DNA methylation, but prevents subsequent embryo development in rapeseed and barley. Front Plant Sci. 2015;6:1-17.

11. Bond DM, Baulcombe DC. Small RNAs and heritable epigenetic variation in plants. Trends Cell Biol. 2014;24:100-7.
12. Schlegel R, Belchev I, Kostov K, Atanassova M. Inheritance of high anther culture response in hexaploid wheat, Triticum aestivum L. var. Svilena. Bulg J Agric Sci. 2000;6:261-70.

13. Rubtsova M, Gnad H, Melzer M, Weyen J, Gils M. The auxins centrophenoxine and 2,4-D differ in their effects on non-directly induced chromosome doubling in anther culture of wheat (T. aestivum L.). Plant Biotechnol Rep. 2013;7:247-55

14. Touraev A, Pfosser M, Heberle-Bors E. The Microspore: A Haploid Multipurpose Cell. Adv Bot Res. 2001;35:53-109.

15. Indrianto A, Barinova I, Touraev A, Heberle-Bors E. Tracking individual wheat microspores in vitro: Identification of embryogenic microspores and body axis formation in the embryo. Planta. 2001;212:163-74.

16. Grabherr MG, Haas BJ, Yassour M, Levin JZ, Thompson DA, Amit I, et al. Full-length transcriptome assembly from RNA-Seq data without a reference genome. Nat Biotechnol. 2011;29:644-52.

17. Dvorak J. Molecular Characterization of a Diagnostic DNA Marker for Domesticated Tetraploid Wheat Provides Evidence for Gene Flow from Wild Tetraploid Wheat to Hexaploid Wheat. Mol Biol Evol. 2006;23:1386-96.

18. Conesa A, Götz S, García-Gómez JM, Terol J, Talón M, Robles M. Blast2GO: a universal tool for annotation, visualization and analysis in functional genomics research. Bioinformatics. 2005;21:3674-6.

19. Haas BJ, Papanicolaou A, Yassour M, Grabherr M, Blood PD, Bowden J, et al. De novo transcript sequence reconstruction from RNA-seq using the Trinity platform for reference generation and analysis. Nat Protoc. 2013;8:1494-512.

20. Ensembl Plants. Triticum aestivum cDNA sequences. 2015: ftp://ftp. ensemblgenomes.org/pub/plants/release-26/fasta/triticum_aestivum/cdna/.

21. Köszegi D, Johnston AJ, Rutten T, Czihal A, Altschmied L, Kumlehn J, et al. Members of the RKD transcription factor family induce an egg cell-like gene expression program. Plant J. 2011;67:280-91.

22. Tsuwamoto R, Yokoi S, Takahata Y. Arabidopsis Embryomaker encoding an AP2 domain transcription factor plays a key role in developmental change from vegetative to embryonic phase. Plant Mol Biol. 2010;73:481-92.

23. Zhou Y, Tan B, Luo M, Li Y, Liu C, Chen C, et al. Histone Deacetylase19 Interacts with HSL1 and Participates in the Repression of Seed Maturation Genes in Arabidopsis Seedlings. Plant Cell. 2013;25:134-48.

24. Nagasaki H, Itoh J, Hayashi K, Hibara K, Satoh-Nagasawa N, Nosaka M, et al. The small interfering RNA production pathway is required for shoot meristem initiation in rice. PNAS. 2007:104:14867-71.

25. Solís MT, Rodríguez-Serrano M, Meijón M, Cañal MJ, Cifuentes A, Risueño $M C$, et al. DNA methylation dynamics and MET1a-like gene expression changes during stress-induced pollen reprogramming to embryogenesis. J Exp Bot. 2012;63:6431-44

26. Muñoz-Amatriaín M, Svensson JT, Castillo AM, Cistué L, Close TJ, Vallés MP. Transcriptome analysis of barley anthers: Effect of mannitol treatment on microspore embryogenesis. Physiol Plant. 2006;127:551-60.

27. Tanaka M, Kikuchi A, Kamada H. The Arabidopsis Histone Deacetylases HDA6 and HDA19 Contribute to the Repression of Embryonic Properties after Germination. Plant Physiol. 2007;146:149-61.

28. Wakui K, Takahata Y. Isolation and expression of Lea gene in desiccationtolerant microspore-derived embryos in Brassica spp. Physiol Plant. 2002; 116:223-30.

29. Colcombet J, Boisson-Dernier A, Ros-Palau R, Vera CE, Schroeder J. Arabidopsis Somatic Embryogenesis Receptor Kinases 1 and 2 are essential for tapetum development and microspore maturation. Plant Cell. 2005;17: 3350-61.

30. Corral-Martínez P, Parra-Vega V, Seguí-Simarro JM. Novel features of Brassica napus embryogenic microspores revealed by high pressure freezing and freeze substitution: evidence for massive autophagy and excretion-based cytoplasmic cleaning. J Exp Biol. 2013;64:3061-75.

31. Jacquard C, Mazeyrat-Gourbeyre F, Devaux P, Boutilier K, Baillieul F, Clement C. Microspore embryogenesis in barley: anther pre-treatment stimulates plant defence gene expression. Planta. 2008;229:393-402.

32. Borges F, Martienssen RA. The expanding world of small RNAs in plants. Nat Rev Mol Cell Biol. 2015;16:727-41.

33. Matsunaga W, Ohama N, Tanabe N, Masuta Y, Masuda S, Mitani N, et al A small RNA mediated regulation of a stress-activated retrotransposon and the tissue specific transposition during the reproductive period in Arabidopsis. Front Plant Sci. 2015;6:48

34. McCue AD, Nuthikattu S, Reeder SH, Slotkin RK. Gene Expression and Stress Response Mediated by the Epigenetic Regulation of a Transposable Element Small RNA. PLoS Genet. 2012;8, e1002474. 
35. Griffiths-Jones S. miRBase: microRNA sequences, targets and gene nomenclature. Nucleic Acids Res. 2006;34:D140-4.

36. Luo YC, Zhou H, Li Y, Chen JY, Yang JH, Chen YQ, et al. Rice embryogenic calli express a unique set of microRNAs, suggesting regulatory roles of microRNAs in plant post-embryogenic development. FEBS Lett. 2006;580: $5111-6$.

37. Zhao B, Liang R, Ge L, Li W, Xiao H, Lin H, et al. Identification of droughtinduced microRNAs in rice. Biochem Biophys Res Commun. 2007;354:585-90.

38. Dong $\mathrm{CH}$, Pei $\mathrm{H}$. Over-expression of miR397 improves plant tolerance to cold stress in Arabidopsis thaliana. J Plant Biol. 2014;57:209-17.

39. Han R, Jian C, LV J, Yan Y, Chi Q, Li Z, et al. Identification and characterization of microRNAs in the flag leaf and developing seed of wheat (Triticum aestivum L.). BMC Genomics. 2014;15:289.

40. Jones-Rhoades MW, Bartel DP, Bartel B. MicroRNAS and their regulatory roles in plants. Annu Rev Plant Biol. 2006:57:19-53.

41. Li H, Soriano M, Cordewener J, Muiño JM, Riksen T, Fukuoka H, et al. The histone deacetylase inhibitor trichostatin a promotes totipotency in the male gametophyte. Plant Cell. 2014;26:195-209.

42. Holding DR, Springer PS. The Arabidopsis gene PROLIFERA is required for proper cytokinesis during seed development. Planta. 2002;214:373-82.

43. Hu X, Zhang Z, Li W, Fu Z, Zhang S, Xu P. cDNA cloning and expression analysis of a putative decarbonylase TaCer1 from wheat (Triticum aestivum L.). Acta Physiol Plant. 2009;31:1111-8.

44. Aarts MG, Keijzer CJ, Stiekema WJ, Pereira A. Molecular characterization of the CER1 gene of arabidopsis involved in epicuticular wax biosynthesis and pollen fertility. Plant Cell. 1995;7:2115-27.

45. Bourdenx B, Bernard A, Domergue F, Pascal S, Leger A, Roby D, et al. Overexpression of Arabidopsis Eceriferum1 Promotes Wax Very-Long-Chain Alkane Biosynthesis and Influences Plant Response to Biotic and Abiotic Stresses. Plant Physiol. 2011;156:29-45.

46. Wang W, Liu X, Gai X, Ren J, Liu X, Cai Y, et al. Cucumis sativus L. WAX2 Plays a Pivotal Role in Wax Biosynthesis, Influencing Pollen Fertility and Plant Biotic and Abiotic Stress Responses. Plant Cell Physiol. 2015;56: 1339-54.

47. Sánchez-Díaz RA, Castillo AM, Vallés MP. Microspore embryogenesis in wheat: New marker genes for early, middle and late stages of embryo development. Plant Reprod. 2013:26:287-96.

48. European Wheat Database. European Cooperative Programme for Plant Genetic Resources. 2016. http://genbank.vurv.cz/ewdb/asp/ewdb_d2. asp?accn=132973. Accessed 24 March 2016

49. Lê Q, Gutièrrez-Marcos JF, Costa LM, Meyer S, Dickinson HG, Lörz H, et al. Construction and screening of subtracted CDNA libraries from limited populations of plant cells: A comparative analysis of gene expression between maize egg cells and central cells. Plant J. 2005:44:167-78.

50. Broad Institute. Picard. Cambridge (MA), USA. http://broadinstitute.github.io/ picard/releases/tag/1.106. (2014).

51. Camacho C, Coulouris G, Avagyan V, Ma N, Papadopoulos J, Bealer K, et al. BLAST+: architecture and applications. BMC Bioinform. 2009;10:421.

52. Langmead B, Trapnell C, Pop M, Salzberg SL. Ultrafast and memory-efficient alignment of short DNA sequences to the human genome. Genome Biol. 2009;10:R25.

53. Bolstad BM, Irizarry R, Astrand M, Speed TP. A comparison of normalization methods for high density oligonucleotide array data based on variance and bias. Bioinformatics. 2003;19:185-93.

54. R Core Team. R: A language and environment for statistical computing. R Foundation for Statistical Computing, Vienna, Austria. 2012. http:// www. R-project. org

55. Benjamini Y, Hochberg Y. Controlling the False Discovery Rate: A Practical and Powerful Approach to Multiple Testing. J Roy Stat Soc (B). 1995;289300.

56. Bertie A. Java Applications for Teaching Statistics. MSOR Connect. 2005;2:78-81.

57. de Hoon MJL, Imoto S, Nolan J, Miyano S. Open Source Clustering Software. Bioinformatics. 2004;20:1453-4

58. Dai X, Zhao PX. psRNATarget: A plant small RNA target analysis server. Nucleic Acids Res. 2011;39 Suppl 2:155-9.

59. Yang X, Li L. miRDeep-P: A computational tool for analyzing the microRNA transcriptome in plants. Bioinformatics. 2011;27:2614-5.

60. IWGSC. A chromosome-based draft sequence of the hexaploid bread wheat (Triticum aestivum) genome. Science. 2014;345:1251788-8.

\section{Submit your next manuscript to BioMed Central and we will help you at every step:}

- We accept pre-submission inquiries

- Our selector tool helps you to find the most relevant journal

- We provide round the clock customer support

- Convenient online submission

- Thorough peer review

- Inclusion in PubMed and all major indexing services

- Maximum visibility for your research

Submit your manuscript at www.biomedcentral.com/submit
Biomed Central 